\title{
Characterization of factor-independent variants derived from TF-1 hematopoietic progenitor cells: the role of the Raf/MAP kinase pathway in the anti-apoptotic effect of GM-CSF
}

\author{
Jhy-Rong Chao ${ }^{1,6}$, Chyi-Shyan Chen ${ }^{1,5}$, Ting-Fang Wang ${ }^{3}$, Li-Hui Tseng ${ }^{4}$, Jaw-Ji Tsai ${ }^{3}$, Min-Liang \\ $\mathrm{Kuo}^{6}$, Jeffrey J-Y Yen ${ }^{2}$ and Hsin-Fang Yang Yen ${ }^{1}$ \\ ${ }^{1}$ Institute of Molecular Biology, and ${ }^{2}$ Institute of Biomedical Sciences, Academia Sinica; ${ }^{3}$ Department of Medicine, Section of \\ Allergy and Clinical Immunology, Cathay General Hospital; ${ }^{4}$ Department of Medical Genetics, National Taiwan University \\ Hospital; ${ }^{5}$ Institute of Biotechnology, Chinese Culture University; ${ }^{6}$ Institute of Toxicology, National Taiwan University Medical \\ School; Taipei, Taiwan, Republic of China
}

\begin{abstract}
Human hematopoietic progenitor cells (TF-1) undergo apoptosis upon deprivation of their dependent cytokine. In this report, we have isolated and characterized some spontaneously derived cytokine-independent variants from TF-1 cells. Analysis of several signaling molecules known to be activated by the GM-CSF pathway revealed that two non-autocrine variants were still responsive to GM-CSF stimulation. However, both variants, without ligand stimulation, already had some activated forms of Raf and MAP kinases. Given current knowledge, the activated Raf/MAP kinase pathway was likely to be responsible for the survival of both variants in the cytokine-free medium. However, the growth of hybrids between wild type and either variant was unexpectedly dependent on GM-CSF. Both variants like the wild type cells were still susceptible to apoptosis induced by other stimuli. These results suggest that either the activated Raf/MAP kinase pathway in both variants is not sufficient to repress the 'two-fold' death signals generated from the hybrids or that there is another mechanism that is responsible for the factor-independent growth of both variants.
\end{abstract}

Keywords: apoptosis; GM-CSF signalling; Raf-1; MAP kinase

\section{Introduction}

Apoptosis (Wyllie, 1987) is one form of cellular suicide that occurs widely during normal development. This process eliminates unwanted cells in a protective way. Recent evidence suggests that the failure of cells to undergo apoptosis may be involved in the pathogenesis of a variety of human diseases, including cancer, autoimmune diseases, and viral infections (reviewed by Steller, 1995; Thompson, 1995). During hematopoiesis, the differentiation and proliferation of various hematopoietic cells are dependent on certain hematopoietic growth factors. For example, hematopoietic progenitor cells are dependent on one or more colony-

Correspondence: HF Yang-Yen, Institute of Molecular Biology, Academia Sinica, Taipei 11529, Taiwan, Republic of China

Received 19 August 1996; revised 11 October 1996; accepted 14 October 1996 stimulating factors (Metcalf, 1989; Williams et al., 1990; Koury and Bondurant, 1990); T lymphoblasts are interleukin-2 (IL-2)-dependent (Duke and Cohen, 1986). Without these growth factors, the majority of these dependent cells die through apoptosis. It has been recently found that the capacity of human primary leukemic blasts to proliferate autonomously in vitro is associated with highly aggressive acute myeloid leukemia (Lowenberg et al., 1993). This suggests that acquisition of autonomous growth of hematopoietic cells may be one important step leading to leukemogenesis.

GM-CSF and IL-3 are two hematopoietic cytokines produced by activated $\mathrm{T}$ cells and mast cells that are potent growth factors for multipotential hematopoietic progenitors as well as various other hematopoietic cells (Arai et al., 1990). The high affinity human receptors for GM-CSF, IL-3 and another cytokine IL-5 are composed of a shared common $\beta$ subunit ( $\beta$ c subunit) and a distinct $\alpha$ subunit which is specific for each cytokine (Miyajima et al., 1993). While the $\alpha$ subunit determines the specificity of the ligand binding, the $\beta \mathrm{c}$ subunit is largely responsible for signal transduction (Sakamaki et al., 1992). Several intracellular signaling proteins are known to be activated once the GM-CSF/ IL-3 receptor is ligand-activated. These include induction of c-myc and c-fos/c-jun genes, activation of Jak2 kinase and tyrosine phosphorylation of other cellular proteins, and activation of the Ras, Raf, and MAP kinase pathway (Sato et al., 1993; Quelle et al., 1994). Recent studies have shown that the proliferation and the anti-apoptotic function of IL-3/GM-CSF are mediated by two distinct pathways (Kinoshita et al., 1995; Yen et al., 1995). It has been further suggested that the receptor-activated Ras/Raf/MAP kinase pathway is responsible for the anti-apoptotic effect of IL-3/ GM-CSF (Kinoshita et al., 1995).

As a first step to study the link between the autonomous (factor-independent) growth and leukemogenesis of hematopoietic cells, we have isolated and extensively characterized two non-autocrine variants derived from the GM-CSF-dependent, human hematopoietic progenitor cells (TF-1). In this report, we present data showing that either the activated Raf/ MAP kinase pathway in both variants is not sufficient to repress the 'twofold' death signals generated from the hybrids or that there is another mechanism that accounts for the factor-independent growth of these cells. 
A
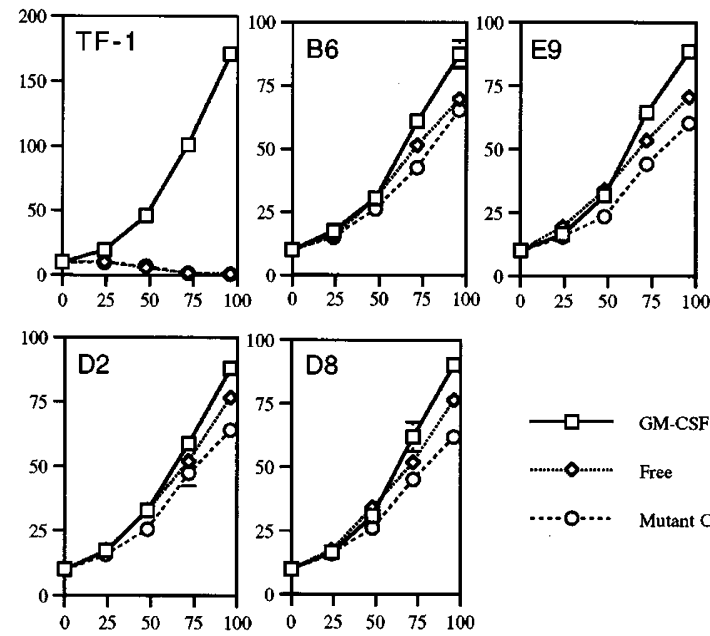

$\mathbf{B}$

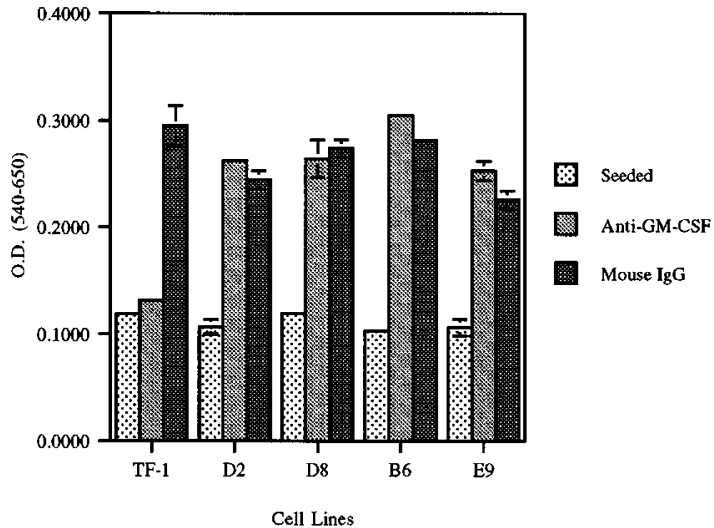

Figure 1 Variant cells proliferate in a cytokine-independent manner. (A) Growth curves of TF-1 and variant cells cultivated in various media as indicated. TF-1 behaved identically in conditioned medium (CM) from each variant. Only the result with $\mathrm{CM}$ from B6 cells was shown panel A labeled TF-1). X and Y axes denote hours after seeding and viable cell numbers $\left(\times 10^{-4}\right)$, respectively. Each point represents the mean value of triplicate experiments. Very similar results were obtained from three other independent experiments. (B) The proliferation of cells cultivated in medium containing anti-GM-CSF or control (mouse IgG) antibody was measured by MTT assay. Each point represents the mean value of triplicate experiments. Seeded: MTT readings from the initially seeded cells

\section{Results}

TF-1 cells undergo apoptosis once their required cytokine (granulocyte-macrophage colony-stimulating factor, GM-CSF or interleukin-3, IL-3) is depleted from their normal growth medium (Yen et al., 1995). By culturing cells $\left(10^{8}\right)$ in media lacking GM-CSF, four spontaneous variants (D2, D8, B6 and E9) were established. Surface marker analysis revealed no evidence that these variants were derived from or differentiated into a different cell lineage (data not shown), and strongly suggested that they were all originated from the same TF-1 cells. However, due to the selection method that was employed in this study, we had no evidence that these four variants were truly independent clones.

In contrast to the wild-type cells, none of these variants underwent apoptosis upon cytokine-withdrawal (see below). Furthermore, under the same cytokine-free conditions they had a comparable DNA synthesis rate as that of the wild type cells maintained in a medium containing GM-CSF (data not shown). These variants, therefore, proliferated well in the absence of GM-CSF. The addition of GM-CSF did not further significantly stimulate their growth (Figure 1). The growth responses of all variants remained the same following one month's culture in GM-CSFcontaining medium (data not shown), suggesting that their genetic alteration(s) which rendered them insensitive to cytokine-withdrawal was very stable. To examine if any of these variants became autocrine growth, the following experiments were carried out. First, we examined if variant cells secreted any growth factors that could support the growth of wild type cells. As shown in Figure 1a, none of the conditioned medium sampled from the culture supernatant of variant cells supported the growth of the wild type cells. These results suggested that neither GM-CSF nor IL-3 was secreted by the variants. To further confirm

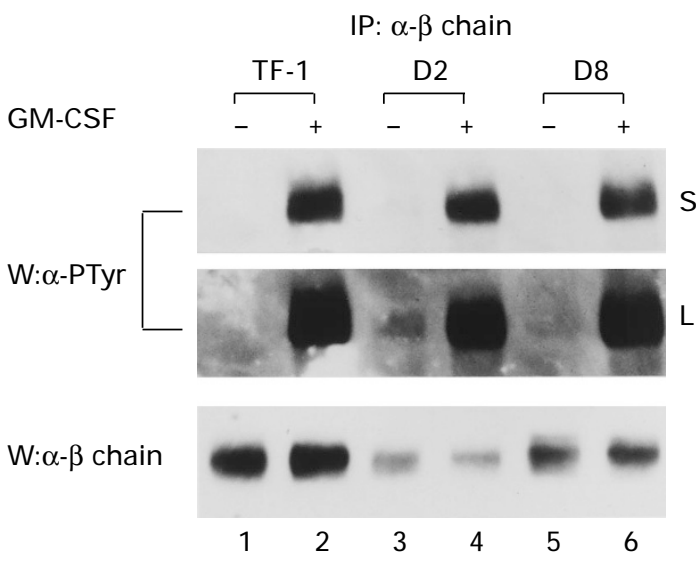

Figure 2 Activation of the GM-CSF receptor $\beta$ chain before and after GM-CSF stimulation. All cells were grown in cytokine-free medium for $20 \mathrm{~h}(-)$ prior to GM-CSF stimulation. Five minutes after stimulation $(+)$, total cell lysates were prepared, immunoprecipitated with antibody specific to receptor $\beta$ chain and the precipitated immunocomplexes were then analysed by immunoblot using anti-phosphotyrosine antibody (upper and middle panels). The same blot was stripped and reprobed with the antibody specific to the receptor $\beta$ chain (lower panel). S and $\mathrm{L}$ denote results from the short and longer exposures, respectively

this, we examined if neutralizing antibody specific to GM-CSF or IL-3 would inhibit the growth of any of these variants. While anti-GM-CSF inhibited the growth of wild-type cells, it had no inhibitory effect on any of the variants (Figure 1b). Similar results were obtained with anti-IL-3 antibody (data not shown). Taken together, these results suggest that the cytokineindependent growth of these variant cells is not due to their endogenous supply of GM-CSF or IL-3.

Another possibility that could generate a factorindependent variant was through the constitutive activation of those signaling molecules that would normally be activated upon ligand binding of the GM- 
CSF receptor. We examined this possibility by checking the activation state of some of these signaling molecules. Due to limit in resources and very similar growth properties between B6 and D2 and between E9 and D8 (data not shown), we only characterized D2 and D8 cells. We first examined if the receptor $\beta$ chain was activated in the absence of GM-CSF. As shown in Figure 2, upon GM-CSF stimulation both variants had similar amounts of tyrosine-phosphorylated receptor $\beta$ chain as the wild type cells even though they both expressed less amounts of the $\beta$-chain protein (compare lanes 2, 4 and 6). However, without ligand stimulation D2 cells had $\sim 1 \%$ of the $\beta$ chain tyrosinephosphorylated (taking the ligand-stimulated amount as $100 \%$ ), while D8 cells had only the background level of activated $\beta$-chain as did the wild-type cells (compare lanes 1, 3 and 5 in the film exposed for a longer time). We next examined if the signalling proteins downstream of the receptor were affected in these two variants. As illustrated in Figure 3, like TF-1 cells D2 and D8 had their Jak2 kinases tyrosine-phosphorylated

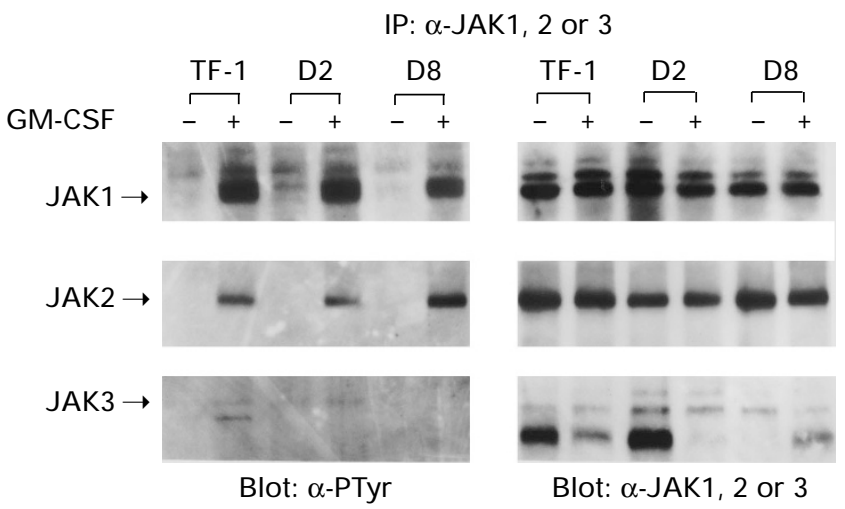

Figure 3 Activation of the JAK family members before and after GM-CSF stimulation. Cells were treated as described in the legend to Figure 2. After stimulation with GM-CSF, cell lysates were prepared from stimulated $(+)$ or untreated $(-)$ cells, and immunoprecipitated with antibodies specific to JAK1, JAK2, or JAK3. The precipitated immunocomplexes were then analysed by immunoblotting with antiphosphotyrosine antibody (left panel). The same blot was stripped, and subsequently blotted with the respective anti-JAK antibody (right panel)
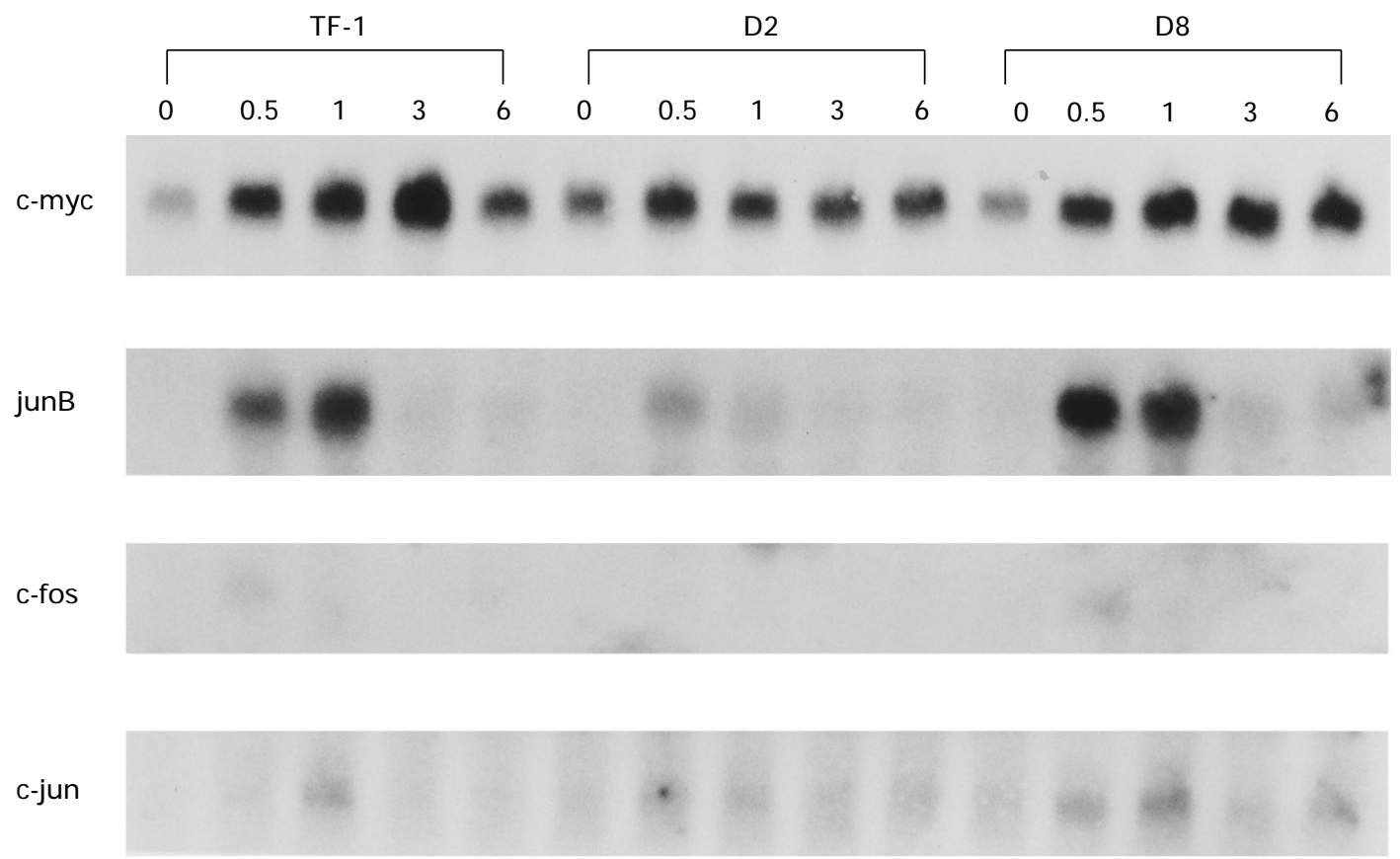

G3PDH
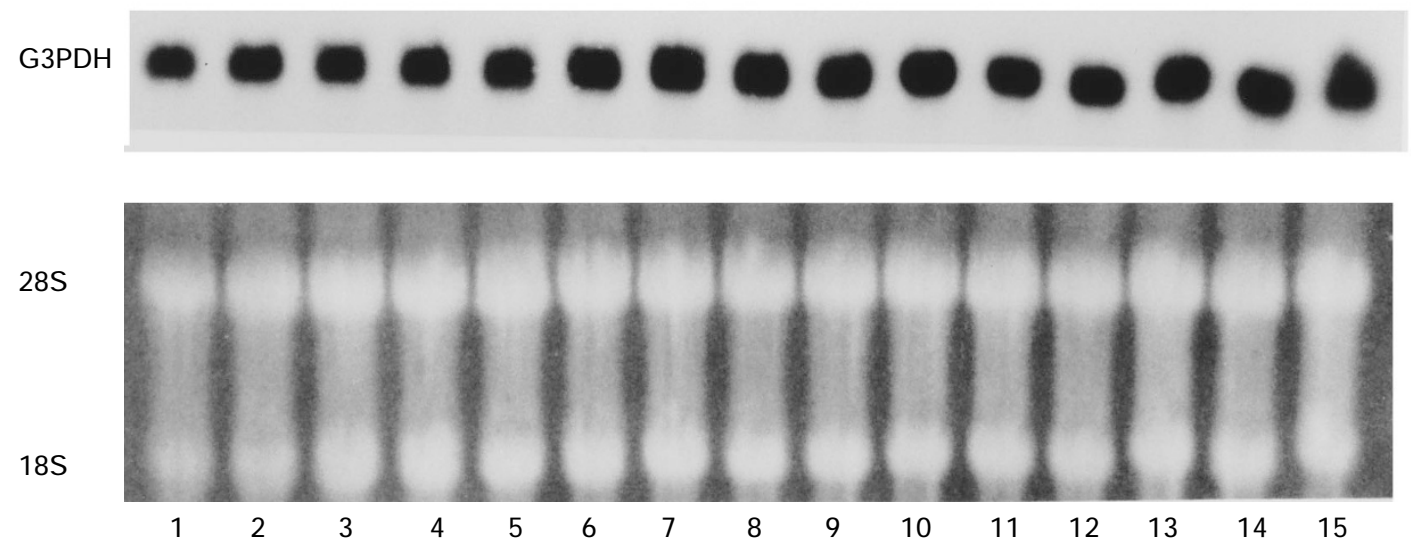

Figure 4 Induction of nuclear proto-oncogenes by GM-CSF in TF-1, D2 and D8 cells. Cells were treated as described in the legend to Figure 2. At various times $(0.5-6 \mathrm{~h})$ after GM-CSF stimulation, total RNAs were prepared and analysed by Northern blotting. The same blot was sequentially probed with ${ }^{32} \mathrm{P}$-labeled cDNA fragments specific to c-myc, Jun B, c-fos, c-jun and G3PDH (glyceraldehyde-3-phosphate dehydrogenase) genes, respectively. The ethidium bromide stained RNA gel (bottom panel) was included as a loading control 

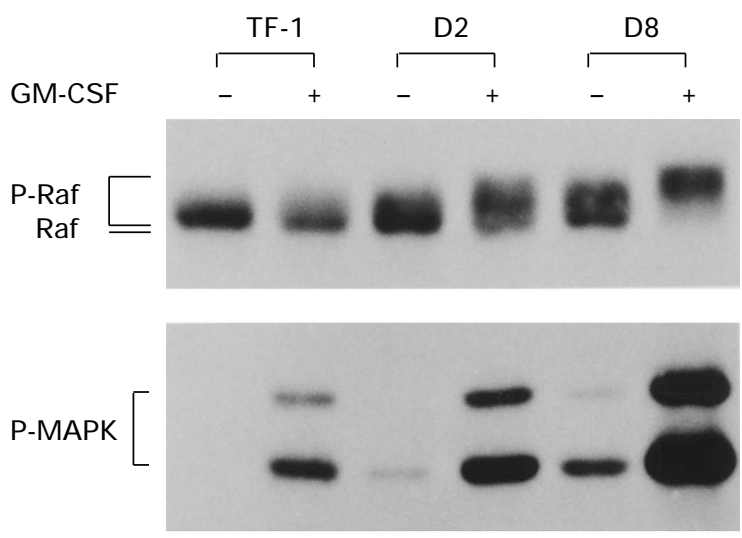

$\alpha$-Tubulin

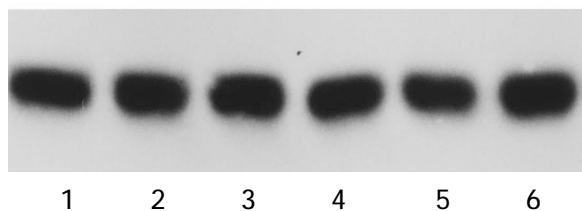

Figure 5 Activation of Raf-1 and MAP I kinases before and after GM-CSF stimulation. Cells shown on top of the figure were treated as described in the legend to Figure 2. Five minutes after stimulation with GM-CSF, cell lysates were prepared from untreated $(-)$ or stimulated $(+)$ cells, and analysed by immunoblot using antibodies specific to Raf-1, phospho-MAP kinase and tubulin, respectively

only upon stimulation with GM-CSF (lanes 2, 4 and 6). Interestingly, in contrast to the negative result reported by (Quelle et al., 1994), we consistently observed the tyrosine-phosphorylation of JAK1 upon GM-CSF stimulation of the TF-1 cells (Figure 3 ). This activation was also observed for D2 and D8 cells. However, in the absence of ligand stimulation only D2 had some activated forms of Jak1 ( $\sim 1 \%$ of the ligandstimulated amount; compare lanes in D2 cells with or without GM-CSF).

We further examined if the immediate early genes known to be activated by the receptor were affected in these two variants. We found that in this case D8 behaved very similar to TF-1 cells with the induction levels and kinetics of c-myc, jun B, c-fos and c-jun by GM-CSF (Figure 4, compares lanes 1-5 to lanes 1115). Surprisingly, these genes were neither activated well by GM-CSF in D2 cells, nor did they express at a higher basal level when cells were depleted of GM-CSF (Figure 4, compare lanes 1 and 6). Next we examined if the Ras/Raf/MAP kinase pathway shown to be important for the anti-apoptotic activity of GM-CSF was affected in these variants. As reported by others (Carroll et al., 1990; Okuda et al., 1992; Welham et al., 1992), GM-CSF also activated Raf-1 and MAP kinases in TF-1 cells (Figure 5). Upon GM-CSF stimulation approximately $30 \%$ of Raf- 1 in TF- 1 cells migrated slower in the SDS-PAGE (lane 2) as indicative of the active forms (Morrison et al., 1988, 1989; Kanakura et al., 1991). However both variants without cytokine stimulation already had large amounts of active forms (D2: $\sim 50 \%$; D8: $>95 \%$; lanes 3 and 5). Stimulation of cells with GM-CSF further increased the percentage of Raf-1 migrated at a much slower position $(>80 \%$ in D2 and $100 \%$ in D8 cells; lanes 4 and 6). Consistent to this result, the MAP kinase, one downstream signalling protein of Raf-1 was also activated accordingly. Upon GM-CSF stimulation, MAP kinases (both p42 and p44 forms) were activated in TF-1 cells (lane 2). These activated forms of MAP kinases could be recognized by a phospho-specific MAP kinase antibody. Both D2 and D8 had some of their MAPKs in the activated forms when they were depleted of GM-CSF (lanes 3 and 5). Again, this activation was more prominent in D8 than in D2 cells. Stimulation of these cells with GM-CSF further increased the percentages of the activated forms (lanes 4 and 6). Taken together, these data suggest that in the absence of GM-CSF the Raf/ MAP kinase pathway is already active in both D2 and D8 cells which may contribute to their survival in the cytokine-free medium.

Besides cytokine-depletion, u.v. irradiation, vaccinia virus infection, and TPA treatments could also induce apoptosis in TF-1 cells (Figure 6a, lane 3; Figure 6b: lanes 3-4; Figure 6c, lane 3). We next examined if these treatments could still induce apoptosis in D2 and D8 cells. As shown in Figure 6, all three treatments induced apoptosis in both variants (Figure 6a: lanes 5 and 7; Figure 6b: lanes 6, 7, 9, 10; Figure 6c: lanes 6, $9)$. However, after TPA treatment the percentage of cells undergoing apoptosis was significantly reduced in D2 but not in D8 cells (Figure 6, compare $44.5 \%$ in D2 to $84.5 \%$ in TF-1 and $78.9 \%$ in D8 cells). The percentage of cells that attached to the culture flasks and underwent differentiation was accordingly increased (Figure 6, compare $55.5 \%$ in D2 to $15.5 \%$ and $21.1 \%$ in TF-1 and D8 cells, respectively).

We next examined if the resistance to cytokinedepletion induced apoptosis of these variants was a dominant or a recessive phenotype. To address this issue, cell fusion experiments were carried out. For this purpose, the wild-type and the variants (D2 and D8) were first engineered to express a dominant selectable marker, neomycin (neo) and hygromycin(hyg) resistant genes, respectively. The resulting clones TF1(neo), D2(hygro) and D8(hygro) (see Materials and methods for generation of these clones) behaved very similar to their parental cells (see below). TF1(neo) were then fused to either D2(hygro) or D9(hygro) cells. The fused cells were selected by their ability to grow in medium containing both G418 and hygromycin. All cell hybrids (either as a single clone or as a mass culture) had twice the DNA contents of the unfused cells, and contained the expected near tetraploid set of chromosomes (data not shown). When these hybrids were grown in medium lacking GM-CSF, they, surprisingly like the wild type cells, underwent apoptosis as manifested by the fragmentation of their genomic DNA (Figure 7). The degree of apoptosis was more prominent in TFD8 than in TFD2 hybrids (Figure 7).

\section{Discussion}

We have isolated and extensively characterized two nonautocrine, factor-independent variants from the GMCSF dependent TF-1 cells. In the absence of GM-CSF, D2 had a minute amount of receptor $\beta$ chain tyrosinephosphorylated and presumably activated. However, this amount of activated $\beta$-chain did not seem to transmit any mitogenic signal further into the nucleus, since its c-myc gene under cytokine-deprivation condi- 


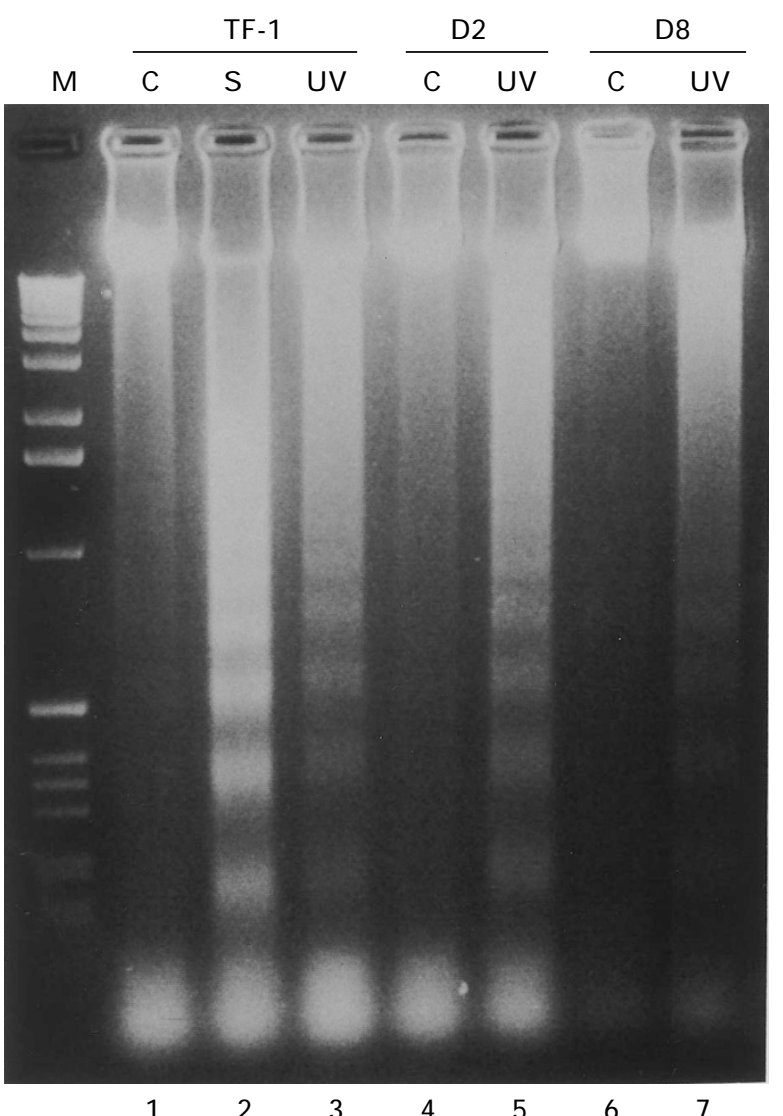

b

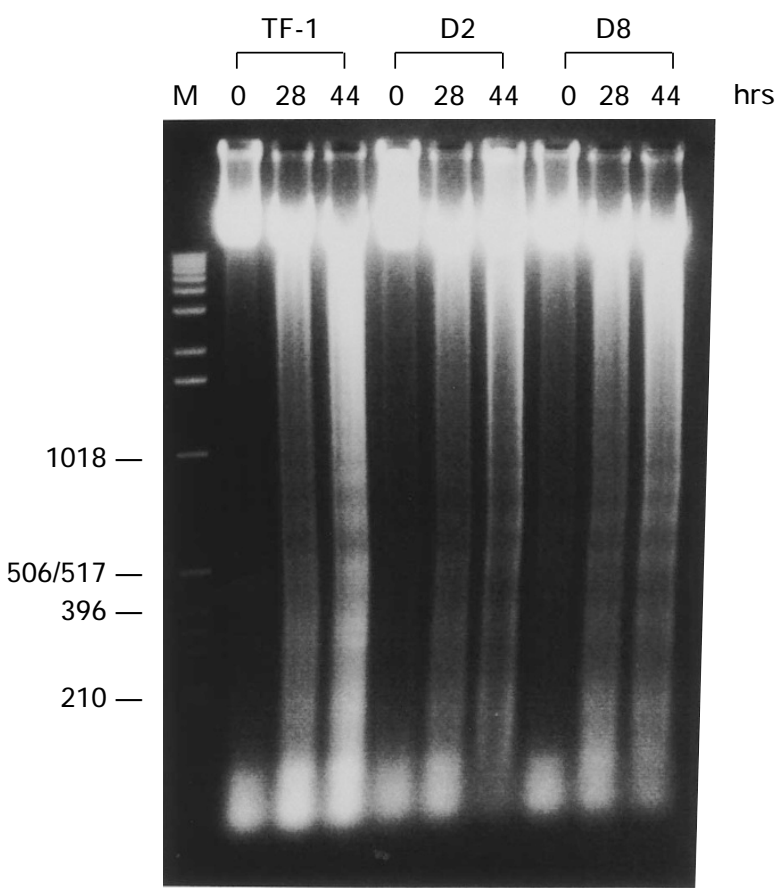

C
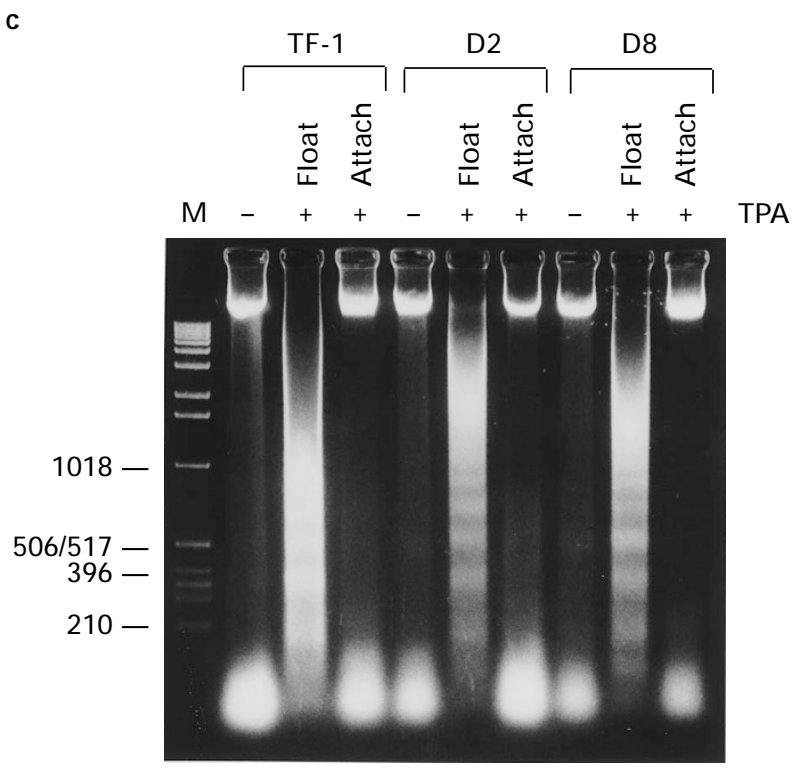

Percentage $\quad 10084.515 .510044 .555 .510078 .921 .1$

Figure 6 Induction of apoptosis by u.v. irradiation, vaccinia virus infection and TPA treatment in TF-1, D2 and D8 cells. (a) Cells cultivated in their respective medium were irradiated with u.v. light $\left(254 \mathrm{~nm}, 0.3 \mathrm{~J} / \mathrm{cm}_{2}\right)$ and recultivated for $4 \mathrm{~h}$ before their genomic DNAs were analysed by $2 \%$ agarose-gel electrophoresis. TF-1 cells deprived of GM-CSF (S, lane 2) were included as a control for cells undergoing apoptosis. Lanes 1, 4 and 6: untreated cells; Lanes 3, 5 and 7: cells irradiated with u.v. light. (b) All cells were individually infected with vaccinia virus at the MO1 of 20 . Twenty-eight or forty-four hours after infection, total genomic DNAs of infected or control cells were analysed by DNA fragmentation assay. (c) Cells in their respective growth medium were each treated with $8 \mathrm{nM}$ of TPA $(+)$ or with vehicle control (-) for $20 \mathrm{~h}$. After treatment, those cells remained in suspension (Float) or attached to the culture flask (Attach) were separately isolated, counted and their genomic DNAs were analysed by DNAfragmentation assay. The percentage of cells in the suspension or attached to the flask was shown on the bottom of the figure. M: $1 \mathrm{~kb}$ DNA marker 
a

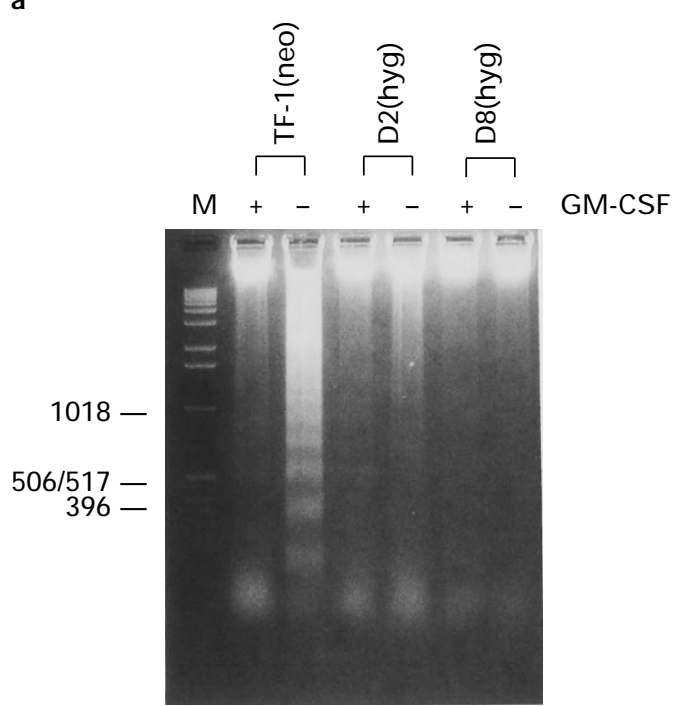

b

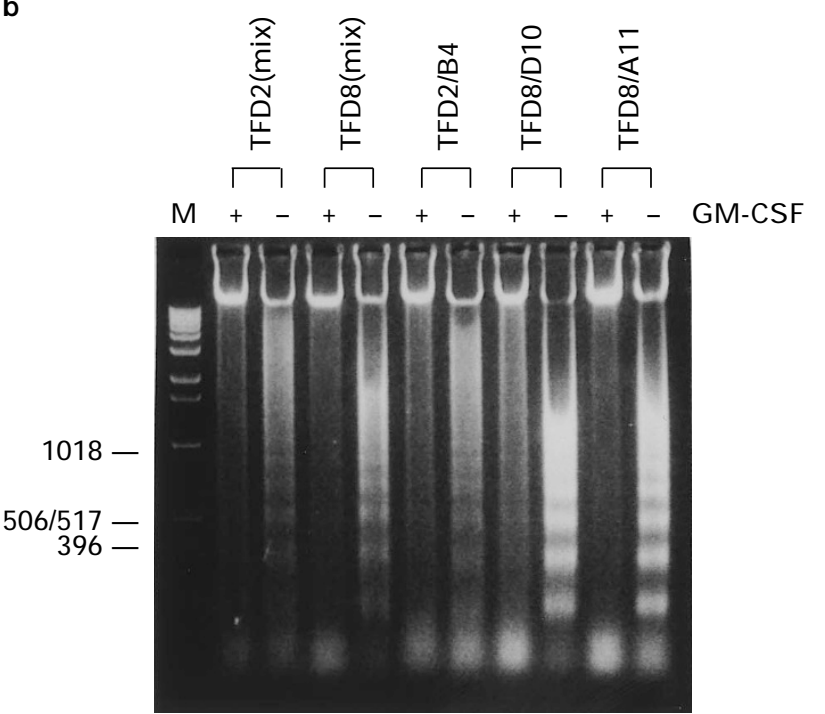

Figure 7 The cell hybrids are susceptible to apoptosis in the absence of GM-CSF. Cells were cultivated in medium with $(+)$ or without (-) GM-CSF. Twenty-four hours after cultivation, the total genomic DNAs of these cells were isolated and analysed by $2 \%$ agarose gel electrophoresis. (a) and (b) show the results obtained from the parental, unfused cells and from the cell hybrids, respectively. M: $1 \mathrm{~kb}$ DNA marker

tion was expressed at a level that was not significantly higher than that of TF-1 cells cultivated under the same conditions. There was a minor amount of Jak 1 activated in the cytokine-depleted D2 cells. This may have resulted from the minute amount of activated $\beta$-chain in these cells. It is not clear if this amount of both proteins would contribute to the factor-independent growth of D2 cells. We did, however, note that D2 but not D8 cells could easily proliferate in the defined medium lacking any serum (J-R Chao, unpublished result). This suggests that the basal activities of both receptor $\beta$ chain and Jak 1 may indeed play some role in the autonomous growth of the D2 cells.

One common element to both D2 and D8 cells was that in the absence of cytokine stimulation both variants had some activated forms of Raf-1 and MAP kinases. The activation of Raf-1 kinase has been noted in a few factor-independent cells either derived spontaneously or by transfection of a cDNA encoding p210 et al., 1994). Furthermore, Kinoshita et al. (1995) reported that the activated Ras/Raf/MAP kinase pathway by the ligand-activated receptor is likely to be responsible for the anti-apoptotic effect of GM-CSF. These results strongly suggest that the activated Raf-1 and MAP kinase observed in both variants may be responsible for their survival in the cytokine free medium. It is not clear what genetic alterations may have activated these two kinases. It may reside in the GM-CSF receptor itself thus activating the Raf/MAP kinase pathway but not other downstream signalling molecules as reported in previous studies (Sato et al., 1993; Inhorn et al., 1995). Alternatively, the alteration may reside in other molecules known to activate the Raf/MAP kinase pathway. Regardless of the cause rendering Raf-1/MAP kinases constitutively active in these cells, this activation does not prevent these two kinases from being further activated by the GM-CSF signalling pathway.

The results of cell fusion were surprising. It showed that even the Raf-1/MAP kinase pathway was active in both variants, their hybrids with the wild-type cells were unexpectedly dependent on GM-CSF for their survival. Several possibilities may explain this result. First, the activated Raf-1/MAP kinase pathway derived from the variants may have been lost in the hybrids. Second, the activated Raf-1/MAP kinases in the hybrids may not be sufficient to inhibit the 'twofold' death signals that would come from both the wild-type and the variant cells upon cytokine deprivation. Third, the observed Raf-1/MAP kinase activation in these variants may not be the causal factor that leads them to be cytokine-independent. Instead, in both variants there may be a loss of function mutation lying close to the death machinery that makes them resistant to cytokine-deprivation induced apoptosis. Upon fusion to the wild type cells the death machinery is restored, and the hybrids subsequently exhibit the wild-type phenotype. Since both variants are still similar to the wild type cells susceptible to other apoptotic stimuli, this loss-offunction mutation, if it exists, is not likely to be the death executor itself. Further experiments are required to determine which possibility may actually account for the phenotype of these two variants.

Although D2 cells are still susceptible to TPA induced apoptosis, the percentage of cells that undergo this process is significantly reduced $(\sim 40 \%$ decrease). It is not clear what other alterations(s) that D2 may have undergone and acquired this phenotype. Wang and Walsh (1996) have recently demonstrated that forced expression of the Cdk inhibitors $\mathrm{p} 21^{\mathrm{CIP} 1}$ or p16 ${ }^{\mathrm{INK} 4 \mathrm{~A}}$ can block apoptosis during myocyte differentiation. It is possible that the extra alteration(s) that D2 cells have acquired is linked to a process that is very similar to the overexpression of some CDK inhibitors in these cells. Alternatively, the decrease in TPA-induced apoptosis in D2 cell may be related to its ability to proliferate in defined medium without cytokine or serum. This additional survival advantage may increase the chance of cells undergoing TPAinduced differentiation. Additional experiments will be required to confirm these hypotheses. 
Materials and methods

\section{Cell culture}

TF-1 cells (Kitamura et al., 1989) were kindly provided by Dr Toshio Kitamura and were maintained in RPMI 1640 supplemented with $10 \%$ fetal bovine serum, $50 \mu \mathrm{M} \beta$ mercaptoethanol, $2 \mathrm{mM}$ L-glutamine, $100 \mu / \mathrm{ml}$ penicillin $\mathrm{G}, 100 \mu \mathrm{g} / \mathrm{ml}$ streptomycin, and $1 \mathrm{ng} / \mathrm{ml}$ of GM-CSF Human GM-CSF was kindly provided by Schering-Plough Ltd, Taiwan. Spontaneous cytokine-independent variants were selected by culturing TF-1 cells in the cytokine-free medium until the vast majority of cells were dead. This process occurred within 3-4 days. After this stage, survival cells were individually cloned by limiting dilution in 96 -well plates. Out of $10^{8}$ cells, four variants were established. These variants (B2, D2, D8 and E9) were maintained in the same medium as that used for wild type cells except that GM-CSF was omitted.

\section{Cell proliferation assay}

Cells were cultured in the indicated growth medium at a density of $1 \times 10^{5}$ cells $/ \mathrm{ml}$. Viable cell numbers were monitored daily by the trypan blue exclusion assay. Proliferation activity was measured by a colorimetric assay with MTT as a substrate, as described by Mosmann (1983). In brief, $2 \times 10^{4}$ cells were seeded in a 96 -well plate and incubated for various times in the indicated medium. MTT $(40 \mu \mathrm{l}$ of $0.8 \mathrm{mg} / \mathrm{ml})$ was added and the MTT reaction was allowed to proceed for $3 \mathrm{~h}$. The purple reaction product was dissolved in $100 \mu \mathrm{l}$ of DMSO and the proliferation activity was expressed as the absorption at $540 \mathrm{~nm}$ minus the background absorption at $650 \mathrm{~nm}$ as measured by a THERMOmax microplate reader (Molecular Devices, Menlo Park, CA).

\section{DNA fragmentation assay}

One million cells were collected following incubation in the desired medium for $24 \mathrm{~h}$ and analysed as previously described (Yen et al., 1995). Briefly, cell pellets were resuspended in $50 \mu \mathrm{l}$ of Williams lysis buffer $(50 \mathrm{~mm}$ TrisHCl, pH 8.0, $10 \mathrm{~mm}$ EDTA, $0.5 \%$ Sarkosyl, and $500 \mu \mathrm{g} / \mathrm{ml}$ of proteinase $\mathrm{K}$ ) and incubated at $50^{\circ} \mathrm{C}$ for $3 \mathrm{~h}$. Ten $\mu$ l of RNase A $(2 \mathrm{mg} / \mathrm{ml})$ was then added to the lysates and the tubes were incubated for an additional hour. This lysate was mixed well with $1 \mu \mathrm{l}$ of ethidium bromide $(20 \mu \mathrm{g} / \mathrm{ml})$, extracted once with an equal volume of phenol-chloroform $(1: 1)$, and stored at $4^{\circ} \mathrm{C}$ after the addition of $20 \mu \mathrm{l}$ of $1 \%$ low melting agarose solution containing $10 \mathrm{~mm}$ EDTA (pH 8.0). Samples were melted at $70^{\circ} \mathrm{C}$ and allowed to solidify inside the well before electrophoresis was initiated.

\section{Northern blotting}

Total RNA was isolated from cultured cells using a method previously described (Liu et al., 1995). Twenty $\mu \mathrm{g}$ of total RNA were resolved on a $1 \%$ formaldehyde-agarose gel and blotted onto a nitrocellulose filter using standard procedure. The blot was then probed sequentially with various $\left[\alpha-{ }^{32} \mathrm{P}\right] \mathrm{dCTP}-\mathrm{labeled}$ DNA fragments labeled to a specific activity of $1 \times 10^{9} \mathrm{cpm} / \mu \mathrm{g}$ using random-priming method. After overnight hybridization at $42^{\circ} \mathrm{C}$ using standard buffer containing $50 \%$ formamide, the blot was washed once in $2 \times \mathrm{SSC}+0.1 \% \mathrm{SDS}$, twice in $0.2 \times \mathrm{SSC}+0.1 \% \mathrm{SDS}$ at $55^{\circ} \mathrm{C}$ and subjected to either autoradiography or direct quantitation using a phosphoImager.
Antibodies

Antibodies specific to JAK1, JAK2 and JAK3 were purchased from Santa Crutz Biotechnology; Anti-phosphotyrosine (clone 4G10) and anti-Raf-1 were from Upstate Biotechnology Incorporated, and Transduction Lab, respectively. Anti-phosph-specific MAPK was obtained from New England Biolabs.

\section{Immunoprecipitation and Western blotting}

Cells to be analysed were lysed in a buffer containing $20 \mathrm{~mm}$ Tris- $\mathrm{HCl}, \mathrm{pH} 7.4,137 \mathrm{~mm} \mathrm{NaCl}, 10 \%$ glycerol, $1 \%$ NP40, $2 \mathrm{mM}$ sodium vanadate and $100 \mathrm{mM}$ sodium fluoride. Following lysis, cell extracts were precleared with protein A-sepharose and immunoprecipitated with a specific, primary antibody. The immunocomplexes were then resolved on a SDS-containing $8 \%$ polyacrylamide gel, transferred to polyvinylidene difluoride nylon membranes (Millipore), and analysed by probing the membrane either with anti-phosphotyrosine antibody or with antibodies specified in the individual experiment. The specific bands were then visualized by ECL (enhanced chemiluminescence) Western blot system (Amersham).

\section{Cell fusion}

Cells to be fused were first engineered to express a selectable marker (neomycin or hygromycin resistant gene) by infecting TF-1 or variants with retroviruses released from the PA317 packaging cells stably transfected with the pBABENeo or pBABEHygro retroviral vector (Morgenstem and Land, 1990). The neomycin-resistant TF1 and the hygromycin-resistant D2 and D8 cells were generated and designated as $\mathrm{TF}-1(\mathrm{Neo})$, D2(Hygro) and D8(Hygro), respectively. These cells were used as a mass culture of drug resistant pool. Fusion was subsequently carried out as follows. Briefly, one million of TF-1(Neo) and one million of D2(Hygro) or D8(Hygro) were pelleted together by centrifugation at 1000 r.p.m. for $5 \mathrm{~min}$. The pellet was gently resuspended in $0.5 \mathrm{ml}$ of polyethelene glycol (PEG, Merck PEG4000) for $2 \mathrm{~min}$, diluted with one $\mathrm{ml}$ of RPMI for $5 \mathrm{~min}$, then $5 \mathrm{ml}$ of the same medium were added and the cells were centrifuged and resuspended in RPMI 1640 containing $10 \%$ and $1 \mathrm{ng} / \mathrm{ml}$ of GM-CSF7. Forty-eight hours later, G418 $(500 \mu \mathrm{g} / \mathrm{ml}$, GIBCO-BRL $)$ and hygromycin $(400 \mu \mathrm{g} / \mathrm{ml}$, Calbiochem) were added to the cells to select the fused populations. These fused cells were analysed as a mass cell culture immediately after drug selection or as a single clone as indicated in the text. TFD2 (mix) and TFD8 (mix) are the mass cell cultures derived by fusing TF-1(neo) with D2(hyr) or with D8 (hyg), respectively. TFD2/B4, TFD8/D10, and TFD8/A11 are single clones selected from TFD2 (mix) or TFD8 (mix), respectively.

\section{Acknowledgements}

We would like to thank Dr Wen Chang for her gift of vaccinia virus, and $\mathrm{Dr} H$ Land for $p$ BabeNeo and pBabeHygro plasmids. This work was supported in part by intramural funding from the Academia Sinica and grants from the National Science Council of Taiwan (NSC83-0203-B-001-002 \& NSC-85-2311-B-001-043, awarded to H-F Y-Y.). 


\section{References}

Arai K, Lee F, Miyajima A, Arai N and Tokota T. (1990). Annu. Rev. Biochem., 59, $783-836$.

Carroll MP, Clark LI, Rapp UR and Mays WS. (1990). J. Biol. Chem., 265, $19812-19817$.

Duke RC and Cohen JJ. (1986). Lymphokine Res., 5, 289 299.

Inhorn RC, Carlesso N, Durstin M, Frank DA and Griffin JD. (1995). Proc. Natl. Acad. Sci. USA, 92, 8665-8669.

Kanakura Y, Druker B, Wood KW, Mamon HJ, Okuda K, Roberts TM and Griffin JD. (1991). Blood, 77, 243-248.

Kinoshita T, Yokota T, Arai K and Miyajima A. (1995). EMBO J., 14, 266-275.

Kitamura T, Tange T, Terasawa T, Chiba S, Kuwaki T, Miyagawa K, Piao YF, Miyazono K, Urabe A and Takaku F. (1989). J. Cell. Physiol., 140, 323-334.

Koury MJ and Bondurant MC. (1990). Science, 248, $378-$ 381.

Liu JJ, Chao JR, Jiang MC, Ng SY, Yen JJ and Yang-Yen HF. (1995). Mol. Cell. Biol., 15, 3654-3663.

Lowenberg B, Van PW, Touw IP, Delwel R and Santini V. (1993). N. Eng. J. Med., 328, 614-619.

Metcalf D. (1989). Nature, 339, $27-30$.

Miyajima A, Mui AL, Ogorochi T and Sakamaki K. (1993). Blood, 82, 1960 - 1974.

Morgenstern JP and Land H. (1990). Nucleic Acids Res., 18, $3587-3596$.

Mosmann T. (1983). J. Immunol. Methods, 65, 55-63.
Morrison DK, Kaplan DR, Rapp U and Roberts TM. (1988). Proc. Natl. Acad. Sci. USA, 85, 8855-8859.

Morrison DK, Kaplan DR, Escobedo JA, Rapp UR, Roberts TM and Williams LT. (1989). Cell, 58, 649-657.

Okuda K, Matulonis U, Salgia R, Kanakura Y, Druker B and Griffin JD. (1994). Exp. Hematol., 22, $1111-1117$.

Okuda K, Sanghera JS, Pelech SL, Kanakura Y, Hallek M, Griffin JD and Druker BJ. (1992). Blood, 79, 2880-2887.

Quelle FW, Sato N, Witthuhn BA, Inhorn RC, Eder M, Miyajima A, Griffin JD and Ihle JN. (1994). Mol. Cell. Biol., 14, 4335-4341.

Sato N, Sakamaki K, Terada N, Arai K and Miyajima A. (1993). EMBO J., 12, $4181-4189$.

Sakamaki K, Miyajima I, Kitamura T and Miyajima A. (1992). EMBO J., 11, 3541-3549.

Steller H. (1995). Science, 267, $1445-1449$.

Tsujimoto Y and Croce CM. (1986). Proc. Natl. Acad. Sci. $U S A, 83,5214-5218$.

Tompson CB. (1995). Science, 267, 1456-1462.

Wang J and Walsh K. (1996). Science, 273, 359-361.

Welham MJ, Duronio V, Sanghera JS, Pelech SL and Schrader JW. (1992). J. Immunol., 149, 1683-1693.

Williams GT, Smith CA, Spooncer E, Dexter TM and Taylor DR. (1990). Nature, 343, $76-79$.

Wyllie AH. (1987). J. Pathol., 153, 313-316.

Yen JJY, Hsieh YC, Yen CL, Chang CC, Lin S and YangYen HF. (1995). J. Immunol., 154, 2144-2152. 